\title{
Educación inclusiva en México: de la teoría a la práctica
}

Inclusive education in Mexico: from theory to practice

Mg. Luz Areli Mendoza

Méndez

Zul_arelime@hotmail.com

México

Recepción

6/ 05/2018

Revisado $12 / 05 / 2018$

Aceptación 23/08/2018

\section{Resumen}

ctualmente en México, se aborda el tema de $\triangle$ trabajar con la diversidad bajo un enfoque de Leducación inclusiva. Sin embargo, ¿cómo se está llevando a cabo? En el siguiente texto se abordan algunos de los fundamentos legales y normativos de la educación inclusiva, desde la perspectiva de un docente de apoyo, así como una reflexión acerca de lo que nos propone la teoría y lo que sucede en las escuelas.

Palabras clave: educación inclusiva, barreras para el aprendizaje y participación, relevancia, equidad, ajustes razonables, Diseño Universal de Aprendizaje.

\section{Abstract}

t present, there is a discussion in Mexico about
working with diversity focusing on inclusive
education. However, there is a question upon how is it being implemented. From the perspective of a support teacher, this paper approaches to some of the legal and normative basis of inclusive education. Moreover, there is also a reflection on some theoretical propositions and its practical application on schools.

Keywords: inclusive education, barriers to learning and participation, relevance, equity, reasonable accommodation, Universal Learning Design. 


\section{Reflexión sobre la práctica}

Día a día me pregunto qué es lo que hacemos en la escuela. Empiezo a recordar desde que llego a la puerta, saludo a los niños y es ahí donde comienzan a surgir más preguntas: ¿tengo claro qué requiere el niño al que acabo de saludar para que pueda desenvolverse dentro del aula y en la escuela?, ¿conozco las barreras a las que se enfrenta y sé cómo apoyarlo para superarlas?

Cuando ingreso al aula regular a trabajar con todos los alumnos, recuerdo que mi propósito es ofrecerles oportunidades de aprendizaje que sean equitativas, fortalecer valores a través de acciones y situaciones reales que los niños y niñas podrían vivir (o viven). Favorecer además sus habilidades a través de juegos, actividades lúdico-recreativas, actividades escritas, trabajo en equipo, entre otras, para así, procurar que ellos descubran todo lo que pueden lograr y se motiven a continuar estudiando y aprendiendo.

En algunas ocasiones, he trabajado de forma individual con los alumnos y me he preguntado si esto es o no "lo indicado" debido a que el alumno se encuentra fuera de su grupo porque la enseñanza es individualizada. Entonces surge la interrogante: ¿existe el trabajo en pares?, ¿será posible este tipo de trabajo? Esto si consideramos que al alumno se le brinden los apoyos requeridos, se realicen los ajustes razonables (modificaciones y adaptaciones), pertinentes a la metodología y evaluación del proceso de enseñanza-aprendizaje. Incluso, notificando a los padres de familia y a los docentes los avances de los niños.

En el trayecto a casa al salir de la escuela, surgen otros cuestionamientos: ¿el día de hoy todos los alumnos tuvieron acceso a una educación inclusiva y de calidad? ¿Qué significa “educación de calidad” y quién la define? ¿Cómo participa en este proceso el docente de apoyo (de educación especial)? ¿Qué se puede hacer el día de mañana en la escuela, en la comunidad, para asegurar un ambiente inclusivo? Surge también una pregunta que, generalmente, comparto con mis compañeros: ¿cómo es que hemos llegado hasta donde estamos en cuestión educativa y en qué fase de ese proceso nos encontramos?

A continuación, un breve recorrido por los antecedentes de la educación inclusiva en México, las implicaciones de cada proceso y cómo es que participo, actualmente, en favorecer ambientes inclusivos. Esto, desde mi función como docente de enlace a la educación inclusiva, la cual consiste en dar seguimiento a aquellos alumnos con discapacidad que se encuentran en 
escuelas regulares; estos alumnos anteriormente se encontraban en un Centro de Atención Múltiple de Educación Especial.

\section{Antecedentes de la educación inclusiva en México}

Nuestro país ha llevado un largo proceso en materia de educación especial, el cual se ha encargado de brindar apoyo en educación básica, pasando por diferentes enfoques desde la inserción escolar e integración educativa. Desde 1995 hasta el 2001 (SEP, 2006), las acciones de educación especial están enfocadas en promover la integración educativa. Bajo este enfoque los estudiantes que se incorporan a las escuelas regulares se han adaptado o «asimilado» a la oferta educativa disponible (currículo, valores, normas, entre otros) independientemente de su origen social y cultural, sus capacidades, su lengua o situaciones de vida. Que el sistema educativo ha permanecido inalterable es la razón por la cual las acciones se centran más en la atención individualizada de las necesidades de los estudiantes (UNESCO, 2010); el alumno debe adaptarse a las condiciones del contexto escolar.

Aunque los niños y jóvenes son aceptados en las escuelas regulares y se continúa luchando por la no discriminación e igualdad de oportunidades; se les llega a etiquetar como alumnos con "Necesidades Educativas Especiales (de aquí en adelante NEE)", aunque estas necesidades se refieren a los apoyos ofrecidos. Por lo tanto, al decir "alumnos con NEE" hacemos alusión a un niño o joven con apoyo, además, "con frecuencia este concepto se utiliza como sinónimo de estudiantes con discapacidad o como una nueva categoría; incluso en nuestro país se utilizó para clasificar a los alumnos con necesidades educativas especiales asociadas y no asociadas a una discapacidad" (SEV, 2012). Por lo anterior, se debe tomar en cuenta el significado y sentido de lo que se quiere transmitir al momento de utilizar dicho concepto.

Finalmente, nos encontramos en transición de la integración educativa a la educación inclusiva, definida por la UNESCO (2009), como un proceso orientado a responder a la diversidad de necesidades de todos los estudiantes, incrementando su participación en el aprendizaje, la cultura y las comunidades y reduciendo y eliminando la exclusión en y desde la educación.

Bajo este enfoque, son los contextos y agentes escolares quienes garantizan la participación de todos los alumnos al tomar en cuenta sus características. Las acciones estarán enfocadas en ir transformando la cultura, organización 
escolar y prácticas educativas encaminadas a la equidad educativa, es decir, a minimizar las Barreras para el Aprendizaje y la Participación que "surgen de la interacción entre el alumno y los distintos contextos en los que se desenvuelven las personas, políticas, instituciones, culturas y circunstancias sociales y económicas que afectan su vida" (Orientaciones Generales para el Funcionamiento de los Servicios de Educación Especial en el Estado de Veracruz, de ahora en adelante: Orientaciones, SEV, 2012). En consecuencia, es el contexto el que puede limitar al individuo y no sus propias características. Esta situación permite hacer partícipes a padres de familia, maestros, alumnos y sociedad en general de las posibles actividades. Sin embargo, esto no solo se limita a las personas con discapacidad. Al contrario, se considera que cualquiera de nosotros puede tener dificultades o vulnerabilidades en un contexto específico, entendiendo a la vulnerabilidad como condición de riesgo que padece un individuo, familia o comunidad y que es resultado de la acumulación de desventajas sociales e individuales; una situación que no puede ser superada en forma autónoma y por la cual existe una limitación para incorporarse a las oportunidades de desarrollo (SEP, 2006).

\section{Marco normativo}

En nuestro país, la educación es un derecho fundamental de toda persona. Plasmado en el artículo tercero constitucional que dice "Todo individuo tiene derecho a recibir educación. El Estado -Federación, Estados, Distrito Federal y Municipios-, impartirá educación preescolar, primaria, secundaria y media superior. La educación preescolar, primaria y secundaria conforman la educación básica; esta y la media superior serán obligatorias" además de laica, gratuita y de calidad, por lo que estará basada en el "mejoramiento constante y el máximo logro académico de los educandos” (DOF, 2013).

Lo anterior quiere decir que todo individuo tiene el derecho y oportunidades de estudiar porque el Estado está obligado a promover, garantizar y proteger la educación pública. Los padres de familia deben llevar a sus hijos a que reciban formación académica a la vez que los niños, jóvenes y adultos la emplean para lograr el máximo aprovechamiento. Sin embargo, ¿hay igualdad de condiciones?, ¿cómo llegamos a una equidad educativa?

Lo anterior está dispuesto en diversos documentos legales, desde nuestra Constitución en el artículo tercero donde se garantiza la educación laica, gratuita, obligatoria y de calidad, pasando por la Ley General de Educación 
en su artículo 41 referente a la educación especial, el Acuerdo 711 (DOF, 2013) que emite las reglas de operación del programa de inclusión y equidad educativa, hasta llegar a las Orientaciones (SEV, 2012) que guían el proceso de educación inclusiva a nivel estatal; a su vez, el contenido de estos textos se refleja en el Plan de Estudios 2011 de Educación Básica donde describen ampliamente los doce Principios Pedagógicos que "son condiciones esenciales para la implementación del currículo, la transformación de la práctica docente, el logro de los aprendizajes y la mejora de la calidad educativa" (SEV, 2012).

Finalmente, con relación a la heterogeneidad de los grupos, encontramos el principio número ocho, referido a la diversidad que alude a favorecer la inclusión para atender a la diversidad. Menciona que la educación es un derecho fundamental y una estrategia para ampliar las oportunidades, instrumentar las relaciones interculturales, reducir las desigualdades entre grupos sociales, cerrar brechas e impulsar la equidad. Por lo tanto, al reconocer la diversidad que existe en nuestro país, el sistema educativo hace efectivo este derecho al ofrecer una educación pertinente e inclusiva. Es pertinente porque respeta la cultura y conocimientos que los niños, niñas y adolescentes tienen sobre el mundo, e inclusiva porque trata de ofrecer una equidad educativa al reducir al mínimo prácticas discriminatorias.

\section{Teoría y práctica}

Dentro de la teoría referente a educación inclusiva, retomaremos lo propuesto por la UNESCO y el Diseño Universal de Aprendizaje en relación a cómo las características de la enseñanza y cómo en el contexto escolar y en el aula los alumnos se pueden enfrentar a barreras para el aprendizaje.

De acuerdo con la UNESCO y Ainscow y Booth (2005), la educación inclusiva se caracteriza por los siguientes elementos:

a. El foco de atención no son ciertos grupos de alumnos sino todos los estudiantes. La finalidad de la inclusión es garantizar una educación de calidad para todos, prestando especial atención a aquellas personas o grupos excluidos o en mayor riesgo de ser marginados o de tener rendimientos menores a los esperados, los cuales pueden variar de un país a otro y de una escuela a otra. 
b. Las acciones están orientadas a transformar la cultura, la organización y las prácticas educativas de las escuelas y otros entornos de aprendizaje para dar respuesta a diversidad de necesidades educativas de todo el alumnado. La inclusión se preocupa de identificar las barreras que limitan el acceso y permanencia en la escuela, la participación y el aprendizaje y buscar la mejor manera de eliminarlas o minimizarlas.

c. Los recursos y sistemas de apoyo están disponibles para todas las escuelas y estudiantes que los requieran.

d. Los docentes se responsabilizan del aprendizaje de todos los estudiantes, independientemente de su origen social y cultural y sus características individuales.

e. Implica una transformación de los sistemas educativos y de la educación general basada en la diversidad y no en la homogeneidad: enfoques, currículo, sistemas de evaluación, formación de los docentes. La atención a la diversidad y la inclusión han de ser un eje transversal de las políticas educativas generales y una responsabilidad del conjunto del sistema educativo.

Por su parte, el Diseño Universal para el Aprendizaje ayuda a estar a la altura del reto de la diversidad sugiriendo materiales de instrucción flexibles, técnicas y estrategias que den poder a los educadores para atender y reconocer estas múltiples necesidades (CAST, 2008). Además, orienta a diseñar currículos flexibles que reduzcan las barreras al aprendizaje y proporcionen fuertes apoyos de aprendizaje para alcanzar las necesidades de todos los aprendices. Lo anterior se fundamenta en tres principios: proporcionar múltiples medios de representación (el «qué» del aprendizaje), brindar múltiples medios para la acción y la expresión (el «cómo» del aprendizaje) y ofrecer múltiples medios de compromiso (el «porqué» del aprendizaje).

En el último principio del Diseño Universal de Aprendizaje se estaría hablando de educación inclusiva, ya que, toma en cuenta la motivación e intereses de los alumnos, haciéndolos partícipes en el proceso de enseñanza - aprendizaje. Si bien la teoría es clara, requerimos saber: ¿cuál es la realidad de las escuelas regulares?,¿cómo se está aplicando la política educativa?, ¿podemos afirmar que nuestras escuelas son inclusivas?,¡por qué? 
He tenido la oportunidad de trabajar en el nivel preescolar y primaria (indígena) como docente de enlace a la educación inclusiva; dentro de mis funciones se encontraban el trabajo colaborativo con alumnos, docentes regulares, padres de familia y comunidad. En ambas escuelas se incluyen niños con discapacidad (intelectual, motriz, y múltiple), los cuales permanecen dentro del aula regular. Recordemos que "inclusión" no tiene que ver solo con el acceso de los alumnos y alumnas con discapacidad a las escuelas comunes, sino también, y de manera contundente, con las acciones de eliminar o minimizar las barreras para el aprendizaje y la participación de todo el alumnado (Booth y Ainscow, 2000). Estas barreras pueden estar presentes dentro del contexto social, familiar y escolar; en conjunto, docentes frente a grupo y docente de educación especial han identificado las principales barreras para el aprendizaje y la comunicación. Sin embargo, estas varían de acuerdo al nivel escolar, contexto escolar y social en que se encuentre la escuela.

Al llegar a las escuelas, una de las primeras acciones consistió en informar sobre el servicio, su misión, el enfoque bajo el cual se trabaja, los especialistas con que se contaba y sus funciones. No fue necesario diseñar estrategias de sensibilización para aceptar a los niños con discapacidad, ya que la comunidad escolar, en general, mostró entusiasmo por trabajar en conjunto y lo vieron como una oportunidad para aprender.

El trabajo en las aulas tampoco se limitó a individualizar la enseñanza, sino, al diversificar las estrategias, se toman como referencia los estilos de aprendizaje de los alumnos; el material a utilizar es variable. Las actividades pedagógicas, además de realizarse en el libro y/o cuaderno, también son fuera del aula, en otros ambientes que propician el aprendizaje de todos los alumnos. Como se menciona en el SIRIED, UNESCO (2010) al hablar de educación inclusiva se supone una nueva visión de la educación, en donde el foco de atención son todos los estudiantes; se pone mayor atención a aquellos en riesgo de ser discriminados o de tener rendimientos menores a los esperados.

Planear para los alumnos con discapacidad o para los que estaban en rezago escolar, ejecutar los ajustes razonables necesarios para el resto del grupo; fue una sugerencia de trabajo que se puso en marcha y dio resultados positivos.

En algunas ocasiones, fue necesario el trabajo individual con el alumno, el cual, de acuerdo con Puigdellívol (1998) resulta indicado cuando la situación 
del alumno requiere una disponibilidad por parte del profesor, difícil de compaginar en situaciones de grupo, aunque este sea reducido.

Si a evaluación nos referimos, esta es realizada por el docente, quien toma en cuenta el aprendizaje esperado que se trabajó, a pesar de que pocas veces se les da a conocer el propósito o lo que se espera que aprendan a los alumnos. La evaluación es formativa; los docentes tratan de valorar el desarrollo seguido por los alumnos y darle mayor relevancia al proceso que al resultado o producto. Adecúan la evaluación de acuerdo al contexto escolar y características del alumno; además, es el docente de apoyo quien ajusta el examen de Español y Matemáticas, mientras que los docentes regulares deciden si harán ajustes al examen escrito de las demás asignaturas o valorarán solo tareas, trabajos, exposiciones y/o investigaciones.

Con lo anterior me pregunto si podemos hablar de educación inclusiva y al hacerlo hablar de educación de calidad, es decir, si hay, de acuerdo con el SIRIED (UNESCO, 2010): "relevancia con respecto a los aprendizajes esperados y contenidosabordados; pertinencia enla aplicación de metodologías, elaboración de ajustes razonables y aprovechamiento de recursos; equidad en oportunidades y prácticas no discriminatorias; y eficiencia que se refiere a la participación de todos los estudiantes en la toma de decisiones, cómo intervienen los padres de familia en la toma de decisiones y la atención a la diversidad".

Afirmar que las escuelas son inclusivas implica una gran responsabilidad, ya que, al ser inclusiva, brinda una educación de calidad, involucra a todos los actores educativos (incluyendo a la comunidad) da la oportunidad de aprender de acuerdo a las características y necesidades de la población; responde a la situación actual de su contexto social, minimiza y/o elimina las barreras para el aprendizaje y la participación, se evita el uso de etiquetas así como las actitudes discriminatorias.

Si bien, el proceso de educación inclusiva se encuentra presente, aún falta trabajo por hacer, pues es un proceso en constante revisión y evaluación. En consecuencia, es dinámico y así como las barreras pueden minimizarse, algunas más pueden hacerse presentes a lo largo del ciclo escolar. 


\section{Educación inclusiva en México}

En México se garantiza el derecho a la educación inclusiva en el artículo 41 de la Ley General de Educación (DOF, 2016):

La educación especial tiene como propósito identificar, prevenir y eliminar las barreras que limitan el aprendizaje y la participación plena y efectiva en la sociedad de las personas con discapacidad, con dificultades severas de aprendizaje, de conducta o de comunicación, así como de aquellas con aptitudes sobresalientes. Atenderá a los educandos de manera adecuada a sus propias condiciones, estilos y ritmos de aprendizaje, en un contexto educativo incluyente, que se debe basar en los principios de respeto, equidad, no discriminación, igualdad sustantiva y perspectiva de género (p.12).

Ambas definiciones coinciden en la atención a la diversidad y en la reducción y/o minimización de las barreras para el aprendizaje y la participación. La principal diferencia es el uso de conceptos, puesto que, México emplea el término educación inclusiva, mientras que, en Uruguay, educación integradora, aunque la esencia de la definición es similar; en México, la Educación Especial será la encargada de promover prácticas inclusivas dentro de la escuela regular de educación básica.

La educación especial dentro del sistema educativo mexicano, de acuerdo con la Ley General de Educación, se encarga de incorporar el enfoque de inclusión y brinda orientaciones a padres de familia y docentes que atiendan a alumnos con discapacidad, con dificultades severas de aprendizaje, de comportamiento o de comunicación, o bien con aptitudes sobresalientes. Para ello, se cuenta con Unidades de Servicio de Apoyo a la Educación Regular (USAER) cuya principal función de acuerdo con las Orientaciones (SEV, 2012) es apoyar el proceso de educación inclusiva en instituciones de educación inicial y escuelas de educación básica regular, a través de la orientación, asesoramiento y acompañamiento para prevenir, minimizar y eliminar las barreras del contexto que limitan la participación y el aprendizaje del alumnado. 


\section{Reflexiones finales}

Después de un breve recorrido por los antecedentes de la educación inclusiva en México, conocer sus características y cómo se está aplicando en algunas escuelas de educación básica, podríamos cuestionarnos: ¿formamos parte de una escuela inclusiva?, ¿cómo participamos nosotros en este proceso?, ¿qué acciones de las mencionadas se realizan diariamente dentro y fuera del aula?, ¿por qué?, ¿cuáles debemos empezar a tomar en cuenta?, ¿de qué manera se garantiza que los alumnos tengan una educación de calidad?, ¿cómo impactan nuestras actitudes, acciones y valores en la transformación de la cultura escolar?, ¿cuáles son las principales barreras para el aprendizaje y la participación a la que se enfrentan día con día nuestros alumnos?, ¿hemos modificado el sistema educativo, dejando de ser homogéneo y basándose en la diversidad?, ¿cómo se atiende a la diversidad?

Las interrogantes quedan abiertas para reflexionar acerca de la situación actual de nuestros centros escolares y cómo cada uno de nosotros influye en la cultura y práctica escolar, que, en suma, lleva a influir en la política educativa.

Como lo menciona Blanco (2015), hablar de educación inclusiva: “(...) implica que todos los niños de una determinada comunidad aprendan juntos independientemente de sus condiciones personales, sociales o culturales. Se trata de lograr una escuela en la que no existan "requisitos de entrada" ni mecanismos de selección o discriminación de ningún tipo; una escuela que modifique substancialmente su estructura, funcionamiento y propuesta pedagógica para dar respuesta a las necesidades educativas de todos y cada uno de los niños y niñas, incluidos aquellos que presentan una discapacidad". (p.12).

Para llegar a brindar educación inclusiva, es necesario el trabajo constante dentro y fuera del aula. Debemos analizar constantemente las acciones y los resultados obtenidos, ir reestructurando, de ser necesario, nuestras formas de enseñanza-aprendizaje, analizar nuestra práctica docente, cómo es que definimos el qué y para qué de la educación, ya que, somos nosotros (los docentes) quienes nos encontramos en las aulas concretando el currículum, ajustando metodologías y estrategias para una mejora en los aprendizajes 
(no sólo de los alumnos), pues el crecimiento profesional se inicia desde el análisis de las propias acciones, como diría Paulo Freire: "solo educadores autoritarios niegan la solidaridad entre el acto de educar y el acto de ser educados por los educandos".

\section{Conclusiones}

1. La actividad docente funciona como oportunidad de desarrollo para el escolar, en tanto gestione aprendizajes equitativos de fortalecimiento de valores a través de acciones y situaciones reales, modelados a través de juegos, actividades lúdico-recreativas, actividades escritas, trabajo en equipo de descubrimiento, estudio y aprendizaje.

2. Aunque en México se ha llevado a cabo un largo proceso en materia de educación especial, se observa que no está clara la denominación "alumnos con necesidades especiales"; con frecuencia este concepto se utiliza como sinónimo de estudiantes con discapacidad, incluso para clasificar a los alumnos con necesidades educativas especiales asociadas y no asociadas a una discapacidad".

3. Los niños de una determinada comunidad deben aprender juntos, independientemente de sus condiciones personales, sociales o culturales.

4. La escuela debe ser un centro abierto en la que no existan "requisitos de entrada" ni mecanismos de selección o discriminación de ningún tipo.

5. La escuela debe tener una propuesta pedagógica para dar respuesta a las necesidades educativas de todos y cada uno de los niños y niñas, incluidos aquellos que presentan una discapacidad. 


\section{Referencias}

Administración Nacional de Educación Pública, Consejo de educación inicial y primaria programa escuelas A.PR.EN.D.E.R. (2016). Disponible en http://www.cep.edu.uy/archivos/proyectos/aprender/2borrador aprender.pdf.

ANEP. CEIP. República Oriental del Uruguay (2010): Orientaciones de políticas educativas del Consejo de Educación Inicial y Primaria. Quinquenio 2010-2014. Disponible en http://www.ceip.edu.uy/ archivos/MaterialesEducativos/opeceip.pdf.

ANEP. CEIP. República Oriental del Uruguay. Proyecto de Presupuesto período 2015- 2019. Disponible en (http://www.anep.edu.uy/anep/ phocadownload/Presupuestosyrendiciones/ProyectodePresupuesto Periodo_2015-2019/Tomo\%201\%20-\%20Exposici\%C3\%B3n\%20 de\%20Motivos\%20ANEP.pdf)

Blanco, R. (sin fecha). Hacia una escuela para todos y con todos. Obtenido el 28 de octubre de 2015. Disponible en http://www.innovemosdoc.cl/ diversidad_equidad/investigacion_estudios/hacia_una_escuela.pdf.

Booth y Ainscow. (2000). Índice de Inclusión. Desarrollando el Aprendizaje y la Participación en las Escuelas. Bristol: UNESCO-Centre for Studies on Inclusive Education (CSEI).

CAST (2008). Universal design for learning guidelines version 1.0. Wakefield, MA: Author. Recuperado el 30 de octubre de 2016. Disponible en http://web.uam.es/personal_pdi/stmaria/sarrio/DOCENCIA/ ASIGNATURA $\% 20$ BASES/LECTURAS $\% 20$ ACCESIBLES $\% 20$ Y\%20GUIONES\%20DE\%20TRABAJO/Diseno\%20Universal\%20 de\%20Aprendizaje.pdf.

Diario Oficial de la Federación (2013). ACUERDO número 711 por el que se emiten las Reglas de Operación del Programa para la Inclusión y la Equidad Educativa. México, Distrito Federal, a 28 de diciembre de 2013. 
Diario Oficial de la Federación (2013). Cámara de Diputados del H. Congreso de la Unión, Constitución Política de los Estados Unidos Mexicanos. Constitución publicada en el Diario Oficial de la Federación el 5 de febrero de 1917. Texto Vigente Últimas reformas publicadas DOF $27-$ 12-2013.

Diario Oficial de la Federación (2016). Cámara de Diputados del H. Congreso de la Unión, constitución política de los estados unidos mexicanos. Ley general de educación. Última reforma publicada DOF 01-06-2016.

Puigdellívol, Ignasi (1998). La educación especial en la escuela integrada. Una perspectiva desde la diversidad. España: GRAO.

Senado y la Cámara de Representantes de la República Oriental del Uruguay, Ley N $\mathrm{N}^{\mathrm{1}} 18.437$, Ley General de Educación aprobación publicada D.O. 16 ene/009 - $\mathrm{N}^{\mathrm{o}} 27654$.

SEP (2011). Plan de estudios 2011. Educación Básica: México: D.F.

SEV, (2012). Orientaciones Generales para el Funcionamiento de los Servicios de Educación Especial del Estado de Veracruz. México: D.F.

UNESCO (2010). Sistema Regional de Información Educativa de los Estudiantes con Discapacidad I SIRIED. Propuesta metodológica. Santiago de Chile.

Viera, A., \& Zeballos, Y. (2014). Inclusión educativa en Uruguay: Una revisión posible. Psicología, Conocimiento y Sociedad, 4(2), 237-260. Disponible en: http://revista.psico.edu.uy/ 